\title{
An approach for an automatic fracture detection of skull DICOM images based on neighboring pixels
}

\begin{abstract}
Providing easy access to Picture Archiving and Communications Systems (PACS) of selected image slices based on its diagnosis would be useful to teach medical students and educators and for government policies. This requires a simplified retrieval presenting only the key images to the doctors that has the diagnosis for every study of interest, thus saving doctors time. An automatic detection of diagnosis will help the radiographers in saving time since they consume a lot of time in the process of detecting skull fractures manually and with the automatic annotation of the pathological terms only to the key slices that has findings/diagnosis of the entire image set an efficient retrieval of specific key slices can be achieved. One important abnormality in the skull is its fracture. The proposed research goal concentrates on the automatic detection of normal and abnormal skull images as a part of our work. This paper presents a simple and fast automatic method to detect skull fracture in Digital Imaging and Communications in Medicine (DICOM) to extract the skull bone using histogram based thresholding and with the neighboring pixel connectivity search to identify the fracture. The experimental results of this approach are reliable with high detection rate.
\end{abstract}

Keyword: Fracture detection; Neighbouring pixel; DICOM image; Skull extraction; Thresholding 